\title{
Importance of ivermectin to human onchocerciasis: past, present, and the future
}

This article was published in the following Dove Press journal:

Research and Reports in Tropical Medicine

27 May 2011

Number of times this article has been viewed

\author{
Ed W Cupp' \\ Charles D Mackenzie ${ }^{2}$ \\ Thomas R Unnasch ${ }^{3}$ \\ 'Department of Entomology and \\ Plant Pathology, Auburn University, \\ Auburn, AL, USA; ${ }^{2}$ Department \\ of Pathobiology, Michigan State \\ University, East Lansing, MI, USA; \\ ${ }^{3}$ Department of Global Health, \\ University of South Florida, Tampa, \\ FL, USA
}

\begin{abstract}
Ivermectin (registered for human use as Mectizan ${ }^{\circledR}$ ) was donated by Merck \& Co Inc in 1987 for the treatment and control of human onchocerciasis ("river blindness"). This philanthropic gesture has had a remarkable effect in reducing the incidence and prevalence of this serious ocular and dermatological disease, while changing health system support for millions of people worldwide. Over 800 million doses have been given to more than 80 million people for onchocerciasis during the past 23 years. As a result, onchocerciasis has been significantly reduced in more than 25 countries, transmission has been interrupted in foci in at least 10 countries, and the disease is no longer seen in children in many formerly endemic foci. Recent communications have suggested that the drug's efficacy as the major therapeutic agent for these control and elimination programs may be threatened, but alternative interpretations for suboptimal response/resistance suggest otherwise. Current research needs and control methods by which the public health community in endemic countries may respond to resistance, should it occur in their area, are discussed, along with the continuing importance of this anthelmintic as the mainstay in onchocerciasis control programs.
\end{abstract}

Keywords: Ivermectin, Onchocerca volvulus, river blindness, resistance, African Programme for Onchocerciasis Control, Onchocerciasis Elimination Program for the Americas

\section{Introduction}

Ivermectin (marketed as Mectizan ${ }^{\circledR}$, Merck \& Co Inc, Whitehouse Station, NJ) is an extremely effective and safe drug for mass treatment of onchocerciasis ("river blindness"). ${ }^{1}$ Country and regional programs, notably countries of the former Onchocerciasis Control Programme (OCP), members of the African Programme for Onchocerciasis Control (APOC), and the Onchocerciasis Elimination Program for the Americas (OEPA), rely on ivermectin for control and elimination of the etiological agent, Onchocerca volvulus. For example, more than one hundred million tablets were used to treat onchocerciasis in 2009, with the bulk of these going to Africa (Table 1).

Because of its effectiveness in killing the dermal stage (microfilaria) of the parasite, with minimal associated pathology, Merck \& Co Inc has donated ivermectin for the past 22 years to countries affected by onchocerciasis and requesting assistance (Figure 1). Over 800 million doses have been given to more than 80 million people during that time. As a result, onchocerciasis has been significantly reduced in more than 25 countries, transmission has been interrupted in foci in at least 10 countries, and onchocerciasis is no longer seen in children in many formerly endemic countries. Consequently, ivermectin monotherapy for onchocerciasis has grown tremendously, receiving funding, technical, and logistical support from international public health
Correspondence: EW Cupp

I309 Allen Street, Owensboro,

KY 42303, USA

Tel +l 2709261559

Email cuppedd@auburn.edu
Research and Reports in Tropical Medicine 201 I:2 8I-92

(C) 20II Cupp et al, publisher and licensee Dove Medical Press Ltd. This is an Open Access article

Dovepress

DOI: $10.2147 /$ RTMM.S19477 which permits unrestricted noncommercial use, provided the original work is properly cited. 
Table I Numbers of treatments (pills) approved by the ivermectin donation program (Merck \& Co Inc) for onchocerciasis in 2007-2009a

\begin{tabular}{llll}
\hline Region $^{\text {b }}$ & $\mathbf{2 0 0 7}$ & $\mathbf{2 0 0 8}$ & $\mathbf{2 0 0 9}$ \\
\hline OEPA & 964263 & $74 I 139$ & 579333 \\
APOC & 64577307 & 64264185 & 79477540 \\
Former OCP & 14912621 & 14974180 & 20299428 \\
Yemen & 73800 & 71040 & 64000 \\
Totals & 80527991 & 80050544 & 100420301 \\
\hline
\end{tabular}

Notes: ${ }^{a}$ Donation program began in 1988; bfigures were provided by the Mectizan ${ }^{\circledR}$ Donation Program, Atlanta, Georgia, and reflect an average of three pills per person treated.

Abbreviations: OEPA, Onchocerciasis Elimination Program for the Americas; APOC, African Programme for Onchocerciasis Control; OCP, Onchocerciasis Control Program (West Africa).

entities, donors, and ministries of health. This collective effort has raised the possibility that onchocerciasis might be controlled, transmission interrupted, and the parasite even focally eliminated using ivermectin monotherapy. Currently, there are more than 40 programs administering the drug on a mass basis (Figure 2). Hence, while often placed in the category of a neglected tropical disease, human onchocerciasis has received extensive support by the international health community.
Several recent reports from Ghana have started to question the efficacy of ivermectin, due largely to the presence of infected persons who responded suboptimally to annual treatment. These reports have evoked concern in the donor and scientific communities. Here, we address this issue, and analyze several representative reports of this possible resistance phenomenon. Where appropriate, we provide alternative interpretations along four lines of reasoning using information from the peer-reviewed scientific literature and recent findings by an international technical consultative committee of APOC. We also review the scientific literature to identify critical research targets and treatment strategies that could be employed immediately to protect the efficacy of ivermectin should resistance prove real.

\section{Contribution of ivermectin to tropical medicine}

Ivermectin has changed the face of tropical medicine perhaps more than any other drug in the past century. Aside from its therapeutic value, ivermectin has also changed the way the developed world intercedes to address major infectious diseases in developing countries. The unprecedented donation

\section{Mass drug administration for onchocerciasis}

150000000

112500000

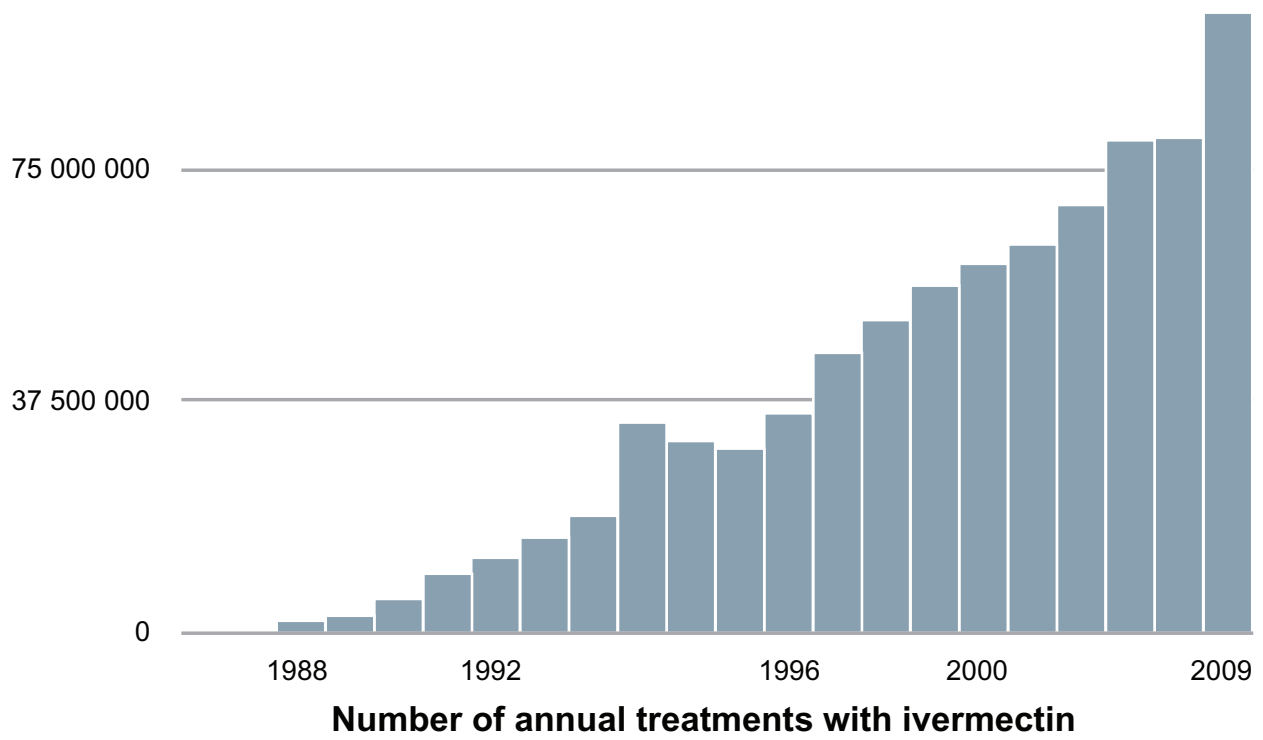

Figure I Donation pattern of ivermectin from the inception of the donation program in 1988-2009. 


\section{Onchocerciasis MDA program number since inception}

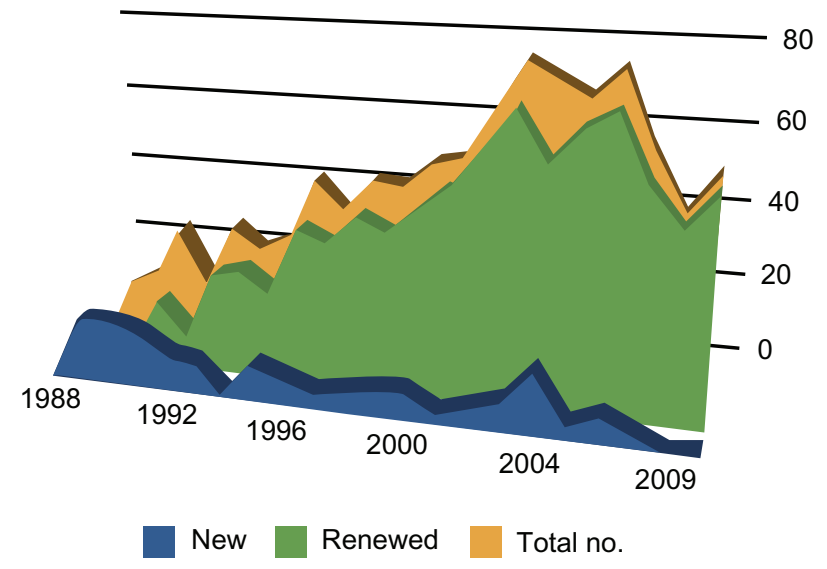

Figure 2 Ivermectin drug administration programs. At the end of 2008, there were 46 programs in operation.

by Merck \& Co Inc of ivermectin for as long as needed to control onchocerciasis ${ }^{2}$ established a standard for other companies to follow, and ushered in major changes in tropical disease control strategies. As a result, mass drug distribution programs were developed that empowered community distributors, which was permissible in part because of the extraordinary safety profile of the drug. ${ }^{3}$ The infrastructure and distribution systems set up for these programs have become a model for health care in many rural areas of Africa and Latin America. The success of the ivermectin-based programs has shown that it is possible to carry out major efforts against chronic diseases in many remote and poor areas of the world, with significant improvements in morbidity, productivity, and longer-term mortality. ${ }^{4}$ Given this success, it is vital for the scientific community to address the issue of potential ivermectin resistance with great care, make the best assessments of the situation, and formulate strategies to address resistance, should it prove to be developing in some parasite populations.

\section{Suboptimal response - is it resistance?}

The idea that drug resistance has occurred in $O$. volvulus is not new. Diethylcarbamazine resistance was suggested to be occurring in 1957 in Mexico, based upon post-treatment survival of a small proportion of skin microfilariae. ${ }^{5}$ This fear proved unfounded when increased regional drug treatments did not lead to development of resistant strains. A suboptimal response to annual ivermectin treatment, defined as "a higher than normal rate of skin repopulation by $O$. volvulus microfilariae" was reported on several occasions in northern
Ghana foci. ${ }^{6,7}$ Surveys carried out as early as 1997 detected individuals with $\geq 10 \mathrm{mf} / \mathrm{snip}$ (microfilariae per skin snip) at 90 and 365 days post-treatment, after purportedly receiving nine or more annual ivermectin treatments. The more rapidly repopulating microfilariae were developmentally competent, ie, they infected vector black flies and developed to infective stage larvae $\left(\mathrm{L}_{3}\right)$, but these progeny parasites remained sensitive to subsequent ivermectin treatment. This suggested that a small proportion of adult female worms had become insensitive to the paralyzing effect of the drug on uterine microfilarial release. This phenomenon was also infrequently observed among ivermectin-naïve individuals, suggesting that it was possibly related to intrinsic parasite factors, ie, drug tolerance. If so, ivermectin-tolerant parasites would be expected to replace drug-sensitive ones slowly and eventually pose a significant public health problem.

Another report from Ghana subsequently suggested that O. volvulus "resistance" to ivermectin was developing. ${ }^{8}$ This conclusion was based on the fact that the prevalence of nonresponders doubled between 2000 and 2005 in two communities. The authors concluded that resistant adult parasite populations were emerging, and that repopulation of the skin with their progeny microfilariae could eventually lead to recrudescence. Several reports questioned this conclusion, suggesting that repopulation of the skin was due simply to failure to achieve adequate drug coverage,, 10 or that young, highly fecund worms were recovering more quickly from treatment. ${ }^{11,12}$ Furthermore, communities in two of the three river basins studied exhibited annual transmission potentials of $\geq 45 \mathrm{~L}_{3}{ }^{6}$ Because transmission of just eight $\mathrm{L}_{3}$ per person per year is considered sufficient to sustain a parasite population (see Table $1^{13}$ ), it is likely that many of the suboptimal responders lived in areas where transmission was at least five times higher than necessary to maintain $O$. volvulus populations. The investigators ${ }^{6}$ contended that an annual transmission potential $\geq 45 \mathrm{~L}_{3}$ would not confound their observations on suboptimal response, citing a figure of $<100$ as an acceptable transmission level. However, the $<100 \mathrm{~L}_{3}$ metric was developed as an indicator of the transmission level below which severe ocular onchocerciasis would not recur, ${ }^{14}$ and is unrelated to population maintenance thresholds.

APOC undertook an indepth analysis of the extent of ivermectin coverage in the affected area in Ghana, ${ }^{15}$ conducting retrospective surveys in 122 villages located within $20 \mathrm{~km}$ (the vector flight range) of the study villages reported by Osei-Atweneboana et al. ${ }^{8}$ The APOC evaluation determined that in areas with normal ("nonresistant") microfilarial skin repopulation rates, all villages had received 
regular annual treatment during the seven years before the Osei-Atweneboana study. ${ }^{8}$ However, in the two areas with rapid microfilarial repopulation, there had been significant coverage problems. In the first, most villages had not been treated at all during the seven years preceding the OseiAtweneboana study. In the second, there were also untreated villages, and annual treatment coverages in the remaining villages were highly inconsistent. ${ }^{15}$ The lack of widespread coverage, and not the emergence of biochemical-based resistance per se, therefore looms as the most likely cause of the observed suboptimal responses. As such, the APOC report is of both practical and theoretical interest, ie, it reiterates the fundamental importance from an operational perspective of achieving adequate coverage and maintaining accurate record-keeping, as well as illustrating the difficulty in attempting to identify and model a phenomenon such as suboptimal response when modeling data are available from a secondary source. ${ }^{16}$

The contribution of the immune response to microfilarial killing and its variation in the human population is another likely contributor to suboptimal response. This was dismissed earlier on the basis that microfilariae from all but two suboptimal responders remained ivermectin-sensitive. ${ }^{6}$ However, it is believed that the immune response is a major contributor to the persisting effect of ivermectin, because this effect extends long after the drug has left the system. Although live nematodes appear to cause minimal inflammation, dying and degenerating parasites do activate such host reactions. This phenomenon was believed to be an explanation for a similar microfilarial repopulation phenomenon reported earlier from the Sudan, where a small proportion of an ivermectin-treated population exhibited a more rapid skin repopulation than expected. ${ }^{17}$ Here, a small group of previously treated persons $(<10 \%$ of total) reported recurrent pruritus, with significantly higher associated loads of dermal microfilariae 4-6 months posttreatment. It was proposed that while microfilarial increase could be attributed to weakening of the paralytic effect of the drug on adult females, it could also reflect an inability of the host's immune system to contribute to drug-initiated microfilarial destruction. In persons lacking the ability to kill microfilariae via an immune response, one could easily overlook this as a possible explanation when there was more rapid skin repopulation, and hypothesize instead that female worms were resistant and better able to release microfilariae. Thus, suboptimal response could be associated with lack of adequate drug coverage or an inability by a few persons to mount a proper immune response.

\section{Conflation of resistance in veterinary parasites with O. volvulus}

Reports discussing possible ivermectin resistance in $O$. volvulus often cite evidence of ivermectin resistance in certain veterinary parasites, particularly Haemonchus contortus, a trichostrongylid nematode parasite of small ruminants. Is this an accurate comparison or simply an example of conflation? Consider that $H$. contortus has a direct life cycle (no intermediate host), normally completes development in about 30 days, has multiple generations a year, and usually exists in focal populations. Ivermectin resistance was first noted in H. contortus in South Africa, where sheep had been dosed at least 26 times in a 33-month period; ${ }^{18}$ under experimental conditions, resistant gene selection by intensive drug treatment and selective inbreeding occurred within a few generations, and quickly became fixed in a small population. ${ }^{19}$ Thus, no one doubts the ability of this species to become quickly resistant to ivermectin (and other drugs) when selection pressure is high and prolonged.

However, the biology of $O$. volvulus is strikingly dissimilar to $H$. contortus, and implies a very different resistance scenario. ${ }^{20} \mathrm{O}$. volvulus has a lengthy prepatent period (12-16 months) and requires a black fly as an intermediate host (vector). The latter serves to broker the infective stage so that the parasite is dependent upon vector survival. Based on field observations, the ratio of surviving $\mathrm{L}_{3} \mathrm{~s}$ to developing larvae $\left(\mathrm{L}_{1}, \mathrm{~L}_{2}\right)$ varies among vector species, but averages roughly $1: 10$, implying that only a small proportion of infected flies survive to become infectious. High vector mortality would thus limit survival of individual $\mathrm{L}_{3}$ carrying resistant alleles. The $\mathrm{L}_{3}$, as the infectious unit, is also passively transported over a fairly large area $\left(\geq 400 \mathrm{~km}^{2}\right.$ in northern Ghana as determined by vector flight range). Gene flow of $O$. volvulus is therefore at least 12 times slower than for $H$. contortus, and the potential breeding population (considered here to be delimited by gene flow within a spatially defined population) encompasses a much larger geographic area, by virtue of vector dispersal. These life cycle features are therefore much more restrictive for selection and fixation of resistant genes than in $H$. contortus.

\section{What vector-transmitted parasites might be harbingers for ivermectin- resistance in $\mathrm{O}$. volvulus?}

Recent controlled laboratory trials suggest that the efficacy of ivermectin in preventing infection with the MP3 strain 
of Dirofilaria immitis ("dog heartworm") has diminished after more than 30 years of use, and therefore this reduction might be confirmatory of a similar phenomenon in O. volvulus. In one study, ${ }^{21}$ one $\operatorname{dog}$ (of 14 in an ivermectin treatment group in which each animal received $50 \mathrm{~L}_{3}$ ) harbored one $D$. immitis adult following a single treatment, while 13/14 dogs in an untreated control group had patent infections, with a geometric mean adult worm count of 22.3. This represented an infection quotient in the ivermectin group of 0.14 (one adult worm from a possible $700 \mathrm{~L}_{3}$, ie, $14 \operatorname{dog} \mathrm{x} \times 50 \mathrm{~L}_{3}$ ). In a second study using the MP3 strain, ${ }^{22}$ seven of eight dogs harbored $D$. immitis adults after being infected with $100 \mathrm{~L}_{3}$ each and later receiving a single treatment with ivermectin, compared with eight of eight dogs with patent infections in an untreated control group. The respective geometric mean worm burden/dog for the ivermectin group was 2.3 adult worms per dog versus 51.6 adult worms per dog in the untreated group, representing a drug efficacy rate of $95.6 \%$ in the ivermectintreated group ( $P=0.0047$ versus untreated controls). The MP3 strain had been isolated in Athens, Georgia in 2006 from a naturally infected dog that was assumed to have never received ivermectin treatment and maintained in the laboratory since that time. A third study ${ }^{23}$ suggested that ivermectin resistance may have occurred in D. immitis populations from Arkansas and Louisiana, based on drug insensitivity of microfilariae as demonstrated by an in vitro test. Insensitivity was positively correlated with selection pressure on a gene encoding a $\mathrm{P}$ glycoprotein.

While the genus Dirofilaria is a sister group phylogenetically related to Onchocerca (and considered to be more closely related than other filariid species such as Wuchereria bancrofti), several important evolutionary and biological disparities exist that impact the rate of drug exposure to sensitive stages, and subsequent selection for resistant forms when attempting to make comparisons between the two species. Among these are different hosts (canine versus human) with different life spans, which have shaped prepatent times of development, ie, development of $D$. immitis requires about six months to reach patency, whereas $O$. volvulus takes 12-16 months. Different sensitivities occur according to developmental stage. The microfilarial, $\mathrm{L}_{3}$, and $\mathrm{L}_{4}$ stages of $D$. immitis are sensitive to drug treatment versus microfilariae and adult worms in $O$. volvulus. Most importantly, the dosing schedule, hence exposure rate, differs because of the desired clinical outcomes. Ivermectin typically is given once a month as prophylaxis for D. immitis infection, whereas the drug is given annually in Africa to resolve dermal and ocular pathology associated with microfilarial O. volvulus infection. Taken collectively, ivermectin exposure to $D$. immitis is approximately $12-24$ times greater than to O. volvulus, ie, 12 treatments per year $\times 2$ generations per year versus 1 treatment per year $\times 1$ generation.

Because of their very similar developmental and phylogenetic histories, the more biologically relevant indicator species are Onchocerca lienalis, Onchocerca gutturosa, Onchocerca gibsoni, and Onchocerca cervicalis. All parasitize domestic livestock, have been exposed to intense ivermectin pressure for more than 25 years on a near-global basis, each has a relatively long life cycle, and each utilizes a blood-sucking fly as intermediate host. $O$. volvulus is considered to have evolved from a bovine antecedent that became adapted to humans within the recent past, ${ }^{24}$ most likely as a result of cattle domestication. ${ }^{24,25}$ In early attempts to find drugs for treatment of human onchocerciasis, parasites from several bovine species were used systematically in both in vivo (O. gibsoni) and in vitro (O. lienalis, O. gutturosa) screens because of the great similarity between the species. Further, the first observation that ivermectin could be useful for treatment of human onchocerciasis without provoking adverse reactions was reported in 1980, after noting that treatment of horses infected with $O$. cervicalis, a common equine parasite, did not result in Mazzotti-type reactions. ${ }^{26}$ Using this breakthrough finding, the first ivermectin trial in humans was then conducted in Africa. Resistance to ivermectin in these veterinary parasites has not been reported, but this issue requires further investigation. Should it be encountered in these particular Onchocerca species, the case for ivermectin resistance in $O$. volvulus becomes much stronger.

\section{Genetic evidence for resistance selection}

The absence of a clearly defined phenotype for ivermectin resistance, lack of a convenient laboratory host for O. volvulus, and the unavailability of tools to manipulate this parasite genetically have made it impossible to conduct studies to associate specific parasite genotypes with resistance directly. Thus, most studies of the development of ivermectin resistance in $O$. volvulus have tended to focus upon allelic frequency changes in certain genes hypothesized to confer resistance. Using this approach, several reports suggest that even short-term ivermectin treatment of $O$. volvulus populations provides sufficient pressure to result in allele frequency shifts. For example, using material from an earlier study, ${ }^{27}$ the effects of genetic selection on $O$. volvulus by 
ivermectin treatment were examined by analyzing changes in the frequencies of three genes, ie, $\beta$-tubulin, heat shock protein 60 , and acidic ribosomal protein, after annual or threemonthly treatments given over a three-year period. ${ }^{28}$ Results indicated a significant selection for $\beta$-tubulin heterozygotes in female worms, with no selection for the other two genes. Allele frequency changes also appeared to occur more rapidly within the three-monthly group. However, there was no selection effect on adult male worms. The effects of selection on fecundity were also evaluated, with the authors concluding that ivermectin selected for females with low fecundity.

The association of an increase in heterozygosity in the $\beta$-tubulin locus was also documented in $O$. volvulus populations in West Africa. ${ }^{29}$ Here, no heterozygotes were detected in a large number of archived parasites collected before the advent of ivermectin treatment, with all parasites containing only a single (A) allele. However, following treatment, an increase in heterozygotes (A/B genotype) was noted. The A/B genotype was also enriched in parasites collected from suboptimal responders. No B/B homozygotes were found, suggesting that the treated population was not in HardyWeinberg equilibrium. However, while a specific polymorphism in the $\beta$-tubulin gene has clearly been associated with the development of benzimidazole resistance in a number of veterinary parasites, ${ }^{30}$ no biochemical rationale exists for associating $\beta$-tubulin with the mechanism of ivermectin action or the development of resistance to the drug.

Similar studies have been carried out looking at other genes that might be associated with the development of ivermectin resistance, including $\mathrm{P}$ glycoprotein ${ }^{31,32}$ and $\mathrm{ABC}$ transporter homologs of $O$. volvulus.$^{33,34}$ Both of these are members of gene families which, in other organisms, encode for pumps capable of excreting multiple drugs from cells. Both studies found changes in allele frequencies associated with ivermectin treatment. Interestingly, in these studies, treatment seemed to result in a homozygote deficit in the treated population, again producing a post-treatment population not in Hardy-Weinberg equilibrium.

However, the significance of these changes in the development of resistance remains unclear, and may result at least in part from genetic bottlenecks resulting from mass drug treatment and disruption of the normal mating behavior of the parasite. For example, a normal nodule usually contains several adult male and female worms, and a single female might be inseminated by more than one male. However, in a nodule from an ivermectin-treated individual, reproduction is often disrupted by limited insemination due to death of male worms. ${ }^{35}$ Together, these effects may disrupt the normal mating process (see Lok et $\mathrm{al}^{36}$ for a similar example), resulting in nonrandom mating. This, in turn, would be expected to result in allele frequency changes (because disruption in mating and reproduction would effectively produce a genetic bottleneck), as well as moving the population away from Hardy-Weinberg equilibrium. Thus, while ivermectin treatment can have dramatic effects upon allele frequencies in $O$. volvulus populations, it is difficult to ascribe these changes solely to the initial stages of resistance selection. Experiments testing the phenotypic effect of the polymorphisms seen to be developing in the $O$. volvulus population, employing a more genetically tractable system, ie, Caenorhabditis elegans, might be useful in answering this question.

It is also possible that genetic variation in the human host may impact drug availability and half-life, thereby affecting the observed response of the parasite population. In this regard, a recent study of human genotypes in Ghana revealed that polymorphisms in the multidrug resistance (MDR1) gene were present at a significantly higher frequency in suboptimal responders than in normal responders, and in random samples of local populations, suggesting that this genetic background could contribute to pharmacokinetic variability and thus the observed suboptimal response. ${ }^{37}$ Several cytochrome P450 haplotypes also varied significantly between optimal responders and suboptimal responders, ${ }^{37}$ indicating their possible role in differential response to ivermectin. Although small numbers of individuals were included in this study, these data point to host genetic differences as an important component in the variable drug response to ivermectin.

\section{The refugium and resistance selection in $\mathbf{O}$. volvulus}

A central feature in blunting genetic selection for resistance in veterinary parasites is the role played by the refugium (ie, "the proportion of the parasite population that is not exposed to a particular given control measure, thus escaping selection for resistance"). ${ }^{38}$ In theory, it is believed that by maintaining a sufficient quantity of susceptible alleles to pair with resistant ones, robust selection for drug resistance can be prevented or decelerated. As a practical matter, the refugium is composed of various untreated subpopulations, which can be stages of parasites in the host not affected by drug treatment, parasites residing in untreated animals, and free-living stages in the environment at the time of treatment. ${ }^{39}$

The example of the role of refugia in preventing selection of ivermectin resistance can be seen in Cyathostominae, the small strongyle nematodes that represent the most prevalent group of horse parasites in countries where anthelmintic 
treatment is routinely employed. The Cyathostominae dwell in the lumen of the large intestine, but the $\mathrm{L}_{3}$ encysts in the mucosa of the gut, serving as a refugium stage. Unlike $H$. contortus, susceptibility to ivermectin has been maintained in the Cyathostominae for over 25 years in the face of intense drug exposure. Only recently have reports of sporadic reduced ivermectin efficacy started to emerge in this group of parasites. ${ }^{40}$

If the concept of the refugium as a way to offset resistance development is applied to the situation in northern Ghana where the suboptimal $O$. volvulus response was described, the reported outcomes are counterintuitive. One would predict that chronically low ivermectin coverage in the "resistant" study areas (as reported by the APOC team) $)^{15}$ would have resulted in a large refugium of untreated infected persons, retarding instead of enhancing development of resistance. Conversely, one would predict that resistance might occur in locations where coverage was consistently high. However, in high coverage villages, microfilarial repopulation rates were within the expected norms. One might argue that underdosing contributed to development of resistance or a suboptimal response. However, several earlier studies, including one in the same region where resistance was reported, had carefully determined the efficacy of ivermectin at the dosage used, and dosage levels in individuals taking the drug were not affected by low coverage rates. ${ }^{41}$ Thus, a perplexing paradox results when the concept of the refugium is invoked as a prophylactic measure in the context of the Ghanaian resistance report.

\section{Recommendations for further action \\ Studies in Pan troglodytes}

To date, there is no direct proof that resistance to ivermectin by $O$. volvulus exists, and the evidence has been indirect, inferential, or correlative. It has been argued that it is difficult to demonstrate ivermectin resistance unequivocally in $O$. volvulus, because this species is an obligate parasite in humans. ${ }^{29}$ However, the chimpanzee (Pan troglodytes) develops patent $O$. volvulus infections, and has proven a useful model for $\operatorname{drug}^{42}$ and pathological ${ }^{43}$ study of the effects of ivermectin treatment on prepatent parasite development, ${ }^{44}$ and studying changes in immune responsiveness over the course of parasite development. ${ }^{45}$ Simple methods are also available to infect chimpanzees with the parasite. ${ }^{46}$ We believe that it is necessary to utilize this model to approach the possibility of ivermectin resistance from a Koch's postulates perspective, ie, to establish or reject firmly the hypothesis of drug resistance in the Ghanaian strains of $O$. volvulus. By using the chimpanzee model in a controlled environment free from confounding effects (such as background transmission), it should be possible to confirm or refute the existence of this phenomenon.

\section{Entomological monitoring and genetics of ivermectin resistance}

To monitor for selection of specific alleles putatively associated with resistance in a breeding population, early detection of resistance is imperative. The $\mathrm{L}_{3}$ is most likely to yield genotypic evidence of resistance soon after mass treatment, ${ }^{47}$ and we suggest that monitoring of $\mathrm{L}_{3}$ be undertaken where resistance is suspected to assess changes in allele frequency and Hardy-Weinberg equilibria of potential resistance loci. These data could be useful in confirming fixation of putative resistant alleles in the suspected population, and can provide an early warning of developing resistance. Conversely, if no evidence of gene selection is detected, this approach would assure control programs that resistance is currently not a threat.

There has been a large number of studies that have employed the free-living nematode $C$. elegans to explore both the mechanism of action of ivermectin and the genetic changes associated with the development of resistance. These studies have suggested that ivermectin acts upon the glutamine-gated chloride channel in C. elegans, ${ }^{48-50}$ and that resistance to ivermectin has been linked with specific changes in the peptides encoded by the avr-14 and avr-15 genes. ${ }^{51}$ Homologs of the avr-14 and avr-15 genes are present in the genome of $O$. volvulus. We recommend that studies be undertaken to determine if alleles corresponding to those experimentally confirmed in C. elegans to confer ivermectin resistance exist in $O$. volvulus, and if evidence for selection of such resistance alleles exists in parasite populations subjected to long-term ivermectin exposure.

\section{Search for a macrofilaricide}

A single ivermectin treatment suppresses microfiladermia for 4-6 months with minimal clinical consequences. Moxidectin, a macrocyclic lactone similar in molecular structure to ivermectin, but with a longer half-life, is currently in Phase III trials. This drug could be more efficacious than ivermectin where annual treatments are given, due to the greater longevity of its effects. However, its registration for future use in the public health arena is uncertain, as is the question of obtaining a donation of the drug similar to that made by Merck in the case of ivermectin. Further, should $O$. volvulus resistance to ivermectin prove real, there is the possibility 
of side resistance to moxidectin because of the structural similarity of the two drugs. ${ }^{20}$ Therefore the development of anthelmintic agents that can quickly and safely destroy the adult worm remains a major goal. Unfortunately, there are few structures that can be considered viable candidates, given the short time frame and long period of drug development usually needed. An oral formulation of flubendazole is currently being considered, because it appears to be differentially effective against adult filariae compared with microfilariae. While still in the developmental stages, research is underway to develop a safe formulation for mass use.

We encourage the continued search for such drugs, but are aware of the challenges associated with gaining approval for a new agent, especially one for mass distribution. Searches to "reposition" old drugs are also encouraged, but should be done with clarity as to their ultimate usefulness, the necessary human margin of safety, and the scale on which the drug would be used. For example, a recent report demonstrated that closantel prevented the $\mathrm{L}_{3}$ to $\mathrm{L}_{4}$ molt of $O$. volvulus by inhibiting chitinase. ${ }^{52}$ O. volvulus $\mathrm{L}_{3}$ molting begins approximately 72 hours postinfection, and requires 5-10 days to complete. In relation to the overall prepatent time course, molting is a "rare event," comprising just 2-3 days of chitin synthesis, so chitinase, the closantel target, would be available for less than 24 hours. Thus, of the 365-425 days required for development, the drug target would be available for $0.7 \%-0.8 \%$ of the time. If one considers the $\mathrm{L}_{4}$ molt to the juvenile stage as representing an equivalent window of availability, then the closantel target would be available for $1.4 \%-1.6 \%$ of the life cycle of the parasite. Molting is also an irregular temporal event, given the mixed pattern of infection within a population. To be useful for mass drug administration, closantel would therefore have to have a long half-life and exhibit a much broader range of activity, preferably also acting on adult worms and/or embryogenesis. Without considering safety, this raises the issue of practicality even if a drug is efficacious. Thus, in the absence of other effective drugs to be used in combination with ivermectin, this drug remains the sole therapy currently available for the control of onchocerciasis.

A significant peripheral problem concerning the use of ivermectin in central Africa for onchocerciasis control is the association of an encephalopathy believed to be caused by killing of Loa loa microfilariae in persons having dual infections. ${ }^{53,54}$ While beyond the scope of this discussion, this clinical syndrome raises important questions as to the utility of ivermectin in core areas where L. loa and $O$. volvulus are coendemic and reinforces the need for a safe and effective macrofilaricide for either parasite species. Such adverse events currently preclude treatment in hypoendemic areas where transmission of onchocerciasis is likely to persist, but where the risk of treatment outweighs the benefit.

\section{New strategies}

Ivermectin is an effective microfilaricidal drug, and when used repetitively on a semiannual basis is also macrofilaricidal. 35,55,56 Twice-yearly treatments in the Americas have proven highly effective where regimens were focused on obtaining $\geq 85 \%$ coverage of eligible persons. This approach, which presumes a refugium of $\leq 15 \%$ of eligible persons, has eliminated significant skin and ocular disease throughout the region and interrupted transmission in seven of the 13 endemic foci after as few as 11 six-monthly treatments (Figure 3 ). ${ }^{57}$ Using this experience as a model, in situations where the type of putative resistance reported from Ghana is believed to occur, twice-yearly treatments with high coverage could resolve the problem. Where possible, integration with lymphatic filariasis control programs would be warranted as well, because long-term biannual treatments have been shown to eliminate $W$. bancrofti. ${ }^{58}$ In this regard, we note that the Ghanaian Ministry of Health has already begun semiannual treatments as a first step to address the suboptimal responder issue.

Annual or six-monthly mass drug administration has also proven successful in interrupting transmission in certain hyperendemic foci in Mali and Senegal following 15-17 years of treatment. ${ }^{59}$ However, to accelerate the march toward elimination, it will be necessary to establish effective guidelines for frequency of future treatments. We believe that treatment regimens should be designed objectively as to frequency, ie, annual versus multiple treatments per year, based on sound epidemiological, biological, and empirical information.

\section{Summary}

The prospect of resistance has caused, not unexpectedly, a level of concern among those responsible for the distribution of ivermectin in the field. It is important that sensible and appropriate information be provided to these stakeholders, include the facts of the situation, their consequences, and how country programs should respond. It is most important to reiterate that ivermectin remains by far the most important weapon in the fight against onchocerciasis, and it is critical 


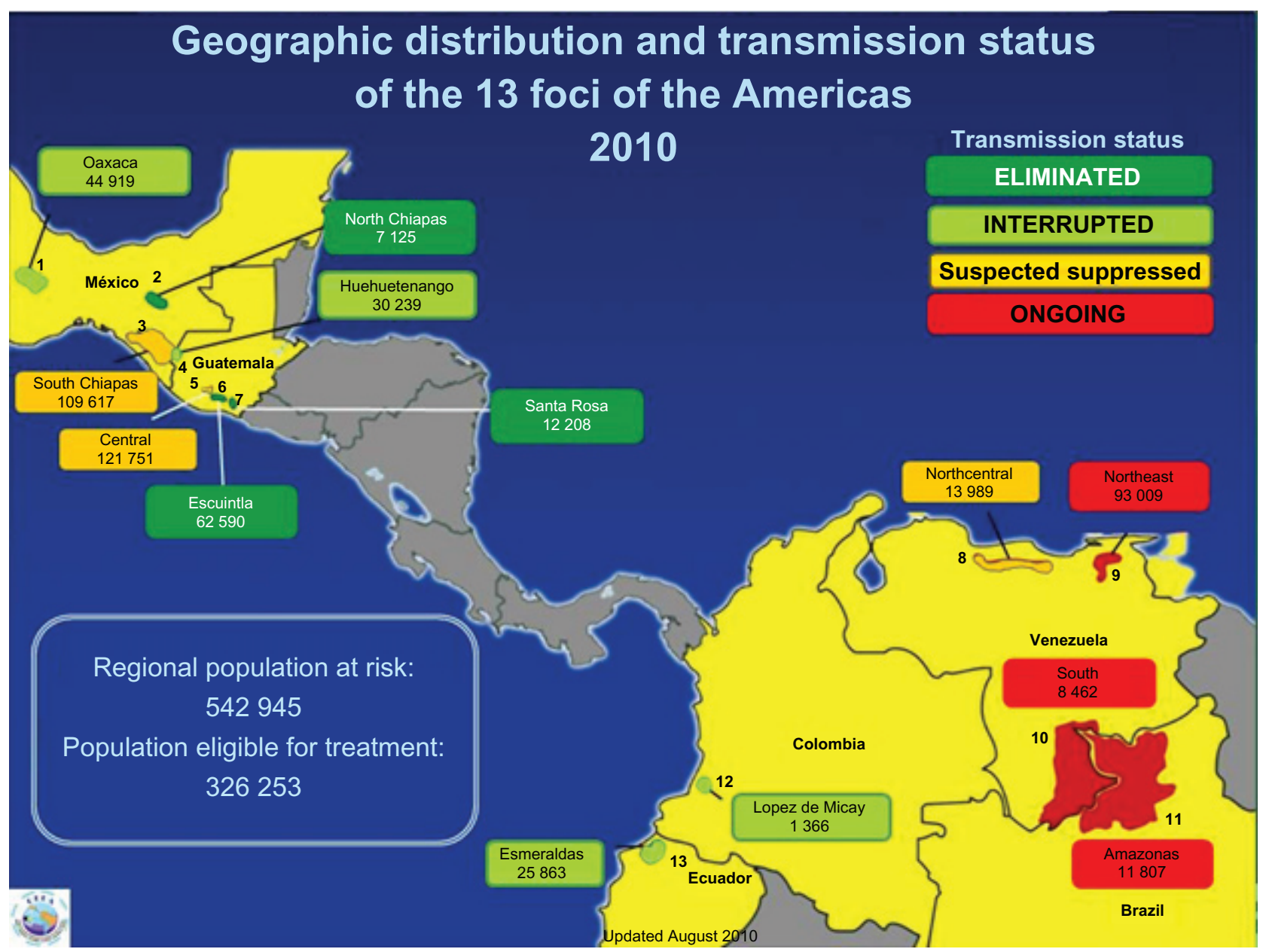

Figure 3 Status of Onchocerca volvulus transmission in the six onchocerciasis elimination programs of the Americas member countries. Transmission has been eliminated or interrupted in seven of the 13 endemic onchocerciasis foci in the Americas.

that guidelines be developed to ensure the continuing efficacy of this valuable drug.

Firstly, it should be acknowledged that ivermectin resistance has occurred in some nematode species, and it may therefore occur in $O$. volvulus. However, the life cycle of $O$. volvulus suggests that it is far less likely to develop resistance than the intestinal helminths. Further, the advent of resistance in one geographic area does not necessarily mean that resistance will appear everywhere, as illustrated by the recent success in eliminating $O$. volvulus transmission in three large hyperendemic foci in Mali and Senegal. ${ }^{59}$ This last point raises two important implications, ie, finding a resistant phenotype by random searching of multiple geographic areas is not likely to be successful, and it is important to act promptly and effectively to eliminate the parasite population in any suspected areas of resistance, so as to prevent the spread of the resistant parasite.
It would be appropriate for the senior management of drug distribution programs to provide guidelines to national programs, both to allay alarm and to provide a practical approach to surveillance for potential resistance. National programs need to receive balanced and clear information as to the low likelihood of resistance and the continuing value of ivermectin in their programs.

How should a program manager detect the presence of potential resistance? This is undoubtedly a difficult challenge, unless careful monitoring of indicators of drug effectiveness is already in place. First, there is a need for a real-time operational method to monitor coverage rates to ensure that rapid repopulation of the skin with microfilariae is associated with genetic selection of a molecular mechanism that directly confers biochemical resistance to drug treatment and is not associated with the scope of drug coverage. If coverage is low and/or discontinuous, there may be a false appearance of resistance or 
nonresponse due to the fact that transmission is ongoing. Entomological monitoring using polymerase chain reaction for detection of infective flies would provide initial evidence of recrudescence. Simple surveys could be used for the recrudescence of clinical signs (eg, pruritus) in place of actual parasitological monitoring; however, this imposes a significant burden on local teams. It would be prudent to develop a set of standard guidelines regarding potential resistance, but it is emphasized that this must be done in a manner that does not cause unnecessary alarm to program managers.

Understanding that there is a low likelihood of resistance is the primary goal in any communication with national programs, and continuing their distribution activities remains a paramount objective. If the evidence suggests that resistance may be occurring in a geographic region, a program should be initiated to contain and remove the parasite population in question by using enhanced therapeutic approaches (eg, increased rounds of ivermectin, nodulectomy) or vector control. Where feasible, selective use of doxycycline to treat persons believed to harbor resistant forms might also be appropriate, ${ }^{60}$ and if the problem is believed to be widespread, this approach could be expanded to treatment at the community level. ${ }^{61}$

\section{Conclusion}

In summary, ivermectin has made an enormous contribution, not only to onchocerciasis control but also to the development of health systems in endemic countries. However, monotherapy for onchocerciasis control (as for any other infectious disease) carries a risk of development of resistance. Continuous efforts must be made to monitor for resistance, and suitable strategies should be developed and implemented to limit its spread if it occurs. New and developing ivermectin-based monotherapy programs to control or eliminate onchocerciasis should scale up as quickly as possible to full coverage to achieve their planned endpoints. Ivermectin remains the most important drug against human filariae and is likely to remain so for the foreseeable future, provided that any suspected areas of potential resistance are managed appropriately and efficiently.

\section{Acknowledgments}

The authors thank Dr James Lok for his helpful comments and suggestions about an earlier version of this paper. We also thank the staff of the Mectizan ${ }^{\circledR}$ donation program for providing numerical data of drug treatments used in Table 1 and Figures 1 and 2, and Dr Mauricio Sauerbrey, Director of OEPA, for providing Figure 3.

\section{Disclosure}

The authors report no conflicts of interest in this work.

\section{References}

1. Cupp EW, Sauerbrey M, Richards F. Elimination of human onchocerciasis: History of progress and current feasibility using ivermectin $\left(\right.$ Mectizan $\left.^{\circledR}\right)$ monotherapy. Acta Trop. August 27, 2010. [Epub ahead of print.]

2. Sturchio JL. The case of ivermectin: Lessons and implications for improving access to care and treatment in developing countries. Community Eye Health. 2001;14(38):22-23.

3. Amazigo UV, Brieger WR, Katabarwa M, et al. The challenges of community-directed treatment with ivermectin (CDTI) within the African Programme for Onchocerciasis Control (APOC). Ann Trop Med Parasitol. 2002;96 Suppl 1:S41-S58.

4. Hotez P, Raff S, Fenwick A, Richards F Jr, Molyneux DH. Recent progress in integrated neglected tropical disease control. Trends Parasitol. 2007;23(11):511-514.

5. Vargas L, Tovar J. Resistance of Onchocerca volvulus microfilariae to diethylcarbamazine. Bull World Health Organ. 1957;16(3): 682-683.

6. Awadzi K, Boakye DA, Edwards G, et al. An investigation of persistent microfilaridermias despite multiple treatments with ivermectin, in two onchocerciasis-endemic foci in Ghana. Ann Trop Med Parasitol. 2004;98(3):231-249.

7. Awadzi K, Attah SK, Addy ET, et al. Thirty-month follow-up of sub-optimal responders to multiple treatments with ivermectin, in two onchocerciasis-endemic foci in Ghana. Ann Trop Med Parasitol. 2004;98(4):359-370.

8. Osei-Atweneboana MY, Eng JK, Boakye DA, Gyapong JO, Prichard RK. Prevalence and intensity of Onchocerca volvulus infection and efficacy of ivermectin in endemic communities in Ghana: A twophase epidemiological study. Lancet. 2007;369(9578): 2021-2029.

9. Cupp E, Richards F, Lammie P, Eberhard M. Efficacy of ivermectin against Onchocerca volvulus in Ghana. Lancet. 2007;370(9593):1123.

10. Mackenzie CD. Efficacy of ivermectin against Onchocerca volvulus in Ghana. Lancet. 2007;370(9593):1123.

11. Remme J, Baker RH, De Sole G, et al. A community trial of ivermectin in the onchocerciasis focus of Asubende, Ghana. I. Effect on the microfilarial reservoir and the transmission of Onchocerca volvulus. Trop Med Parasitol. 1989;40(3):367-374.

12. Remme JH, Amazigo U, Engels D, Barryson A, Yameogo L. Efficacy of ivermectin against Onchocerca volvulus in Ghana. Lancet. 2007;370(9593):1123-1124.

13. Basanez MG, Collins RC, Porter CH, Little MP, Brandling-Bennett D. Transmission intensity and the patterns of Onchocerca volvulus infection in human communities. Am J Trop Med Hyg. 2002;67(6): 669-679.

14. Renz A, Wenk P, Anderson J, Fuglsang H. Studies on the dynamics of transmission of onchocerciasis in a Sudan-savanna area of North Cameroon V. What is a tolerable level of annual transmission potential? Ann Trop Med Parasitol. 1987;81(3):263-274.

15. African Programme for Onchocerciasis Control. Report of the twentysixth session of the Technical Consultative Committee. Ouagadougou: APOC. Available from: http://www.who.int/apoc/about/structure/tcc/ Final_Report_TCC_26_English_23_05_08.pdf. Accessed March 10, 2011.

16. Churcher TS, Pion SD, Osei-Atweneboana MY, et al. Identifying suboptimal responses to ivermectin in the treatment of river blindness. Proc Natl Acad Sci U S A. 2009;106(39):16716-16721.

17. Ali MM, Mukhtar MM, Baraka OZ, Homeida MM, Kheir MM, Mackenzie CD. Immunocompetence may be important in the effectiveness of Mectizan (ivermectin) in the treatment of human onchocerciasis. Acta Trop. 2002;84(1):49-53.

18. Carmichael I, Visser R, Schneider D, Soll M. Haemonchus contortus resistance to ivermectin. J South Afr Vet Assoc. 1987;58(2):93. 
19. Coles GC, Rhodes AC, Wolstenholme AJ. Rapid selection for ivermectin resistance in Haemonchus contortus. Vet Parasitol. 2005;129(3-4): 345-347.

20. Shoop WL. Ivermectin resistance. Parasitol Today. 1993;9(5): 154-159.

21. Snyder DE, Wiseman S, Cruthers LR, Slone RL. Ivermectin and milbemycin oxime in experimental adult heartworm (Dirofilaria immitis) infection of dogs. J Vet Intern Med. 2011;25(1):61-64.

22. Blagburn BL, Dillon AR, Arther RG, Butler JM, Newton JC. Comparative efficacy of four commercially available heartworm preventive products against the MP3 laboratory strain of Dirofilaria immitis. Vet Parasitol. 2011;176(2-3):189-194.

23. Bourguinat C, Keller K, Blagburn B, Schenker R, Geary TG, Prichard RK. Correlation between loss of efficacy of macrocyclic lactone heartworm anthelmintics and P-glycoprotein genotype. Vet Parasitol. 2011;176(4):374-381.

24. Bain O. Le Genre Onchocerca: Hypotheses sur son Evolution et cle Dichotomique des Especes [The genus Onchocerca: hypotheses on its evolution and a dichotomous key to its species]. Annales de Parasitologie. 1981;56:503-526.

25. Cupp EW. Human onchocerciasis: Developmental biology of the parasite. Paper presented at: Onchocerciasis/Filariasis. Proceedings of a Symposium; 1986 April 8-10; Kalamazoo, MI.

26. Klei TR, Torbert BJ, Ochoa R. Efficacy of ivermectin (22,23dihydroavermectin B1) against adult Setaria equina and microfilariae of Onchocerca cervicalis in ponies. J Parasitol. 1980;66(5):859-861.

27. Gardon J, Boussinesq M, Kamgno J, Gardon-Wendel N, Demanga N, Duke BO. Effects of standard and high doses of ivermectin on adult worms of Onchocerca volvulus: A randomised controlled trial. Lancet. 2002;360(9328):203-210.

28. Bourguinat C, Pion SD, Kamgno J, et al. Genetic selection of low fertile Onchocerca volvulus by ivermectin treatment. PLOS Negl Trop Dis. 2007;1(1):e72.

29. Eng JK, Blackhall WJ, Osei-Atweneboana MY, et al. Ivermectin selection on beta-tubulin: Evidence in Onchocerca volvulus and Haemonchus contortus. Mol Biochem Parasitol. 2006;150(2):229-235.

30. Lubega GW, Klein RD, Geary TG, Prichard RK. Haemonchus contortus: The role of two beta-tubulin gene subfamilies in the resistance to benzimidazole anthelmintics. Biochem Pharmacol. 1994;47(9):1705-1715.

31. Ardelli BF, Guerriero SB, Prichard RK. Ivermectin imposes selection pressure on P-glycoprotein from Onchocerca volvulus: Linkage disequilibrium and genotype diversity. Parasitology. 2006;132(Pt 3): 375-386.

32. Bourguinat C, Ardelli BF, Pion SD, et al. P-glycoprotein-like protein, a possible genetic marker for ivermectin resistance selection in Onchocerca volvulus. Mol Biochem Parasitol. 2008;158(2):101-111.

33. Ardelli BF, Prichard RK. Reduced genetic variation of an Onchocerca volvulus $\mathrm{ABC}$ transporter gene following treatment with ivermectin. Trans R Soc Trop Med Hyg. 2007;101(12):1223-1232.

34. Prichard RK, Roulet A. ABC transporters and beta-tubulin in macrocyclic lactone resistance: Prospects for marker development. Parasitology. 2007;134(Pt 8):1123-1132.

35. Cupp EW, Duke BO, Mackenzie CD, et al. The effects of long-term community level treatment with ivermectin (Mectizan) on adult Onchocerca volvulus in Latin America. Am J Trop Med Hyg. 2004; 71(5):602-607.

36. Lok JB, Knight DH, Selavka CM, Eynard J, Zhang Y, Bergman RN. Studies of reproductive competence in male Dirofilaria immitis treated with milbemycin oxime. Trop Med Parasitol. 1995;46(4): 235-240.

37. Kudzi W, Dodoo AN, Mills JJ. Genetic polymorphisms in MDR1, CYP3A4 and CYP3A5 genes in a Ghanaian population: A plausible explanation for altered metabolism of ivermectin in humans? BMC Med Genet. 2010;11:111

38. Van Wyk JA. Refugia - overlooked as perhaps the most potent factor concerning the development of anthelmintic resistance. Onderstepoort $J$ Vet Res. 2001;68(1):55-67.
39. Kaplan RM. Drug resistance in nematodes of veterinary importance: a status report. Trends Parasitol. 2004;20(10):477-481.

40. Traversa D. Antihelminthic resistance in horse cyathostomins in Europe: Current status and future perspectives. Scientific Parasitology. 2010;11:1-6.

41. Awadzi K, Dadzie KY, Shulz-Key H, Haddock DR, Gilles HM, Aziz MA. The chemotherapy of onchocerciasis X. An assessment of four single dose treatment regimes of MK-933 (ivermectin) in human onchocerciasis. Ann Trop Med Parasitol. 1985;79(1):63-78.

42. Duke BO. The effects of some drugs - pentamidine, stibocaptate, Hoechst 33258, F 151, compound "E" and Nifurtimox - on Onchocerca volvulus in chimpanzees. Tropenmed Parasitol. 1977;28(4):447-455.

43. Duke BO. Observations on Onchocerca volvulus in experimentally infected chimpanzees. Tropenmed Parasitol. 1980;31(1):41-54.

44. Taylor HR, Trpis M, Cupp EW, et al. Ivermectin prophylaxis against experimental Onchocerca volvulus infection in chimpanzees. Am JTrop Med Hyg. 1988;39(1):86-90.

45. Lobos E, Weiss N, Karam M, Taylor HR, Ottesen EA, Nutman TB. An immunogenic Onchocerca volvulus antigen: A specific and early marker of infection. Science. 1991;251:1603-1605.

46. Cupp EW, Bernardo MJ, Kiszewski AE, Trpis M, Taylor HR. Large scale production of the vertebrate infective stage (L3) of Onchocerca volvulus (Filarioidea: Onchocercidae). Am JTrop Med Hyg. 1988;38(3): 596-600.

47. Churcher TS, Basanez MG. Density dependence and the spread of anthelmintic resistance. Evolution. 2008;62(3):528-537.

48. Cook A, Aptel N, Portillo V, et al. Caenorhabditis elegans ivermectin receptors regulate locomotor behaviour and are functional orthologues of Haemonchus contortus receptors. Mol Biochem Parasitol. 2006;147(1):118-125.

49. Cully DF, Vassilatis DK, Liu KK, et al. Cloning of an avermectinsensitive glutamate-gated chloride channel from Caenorhabditis elegans. Nature. 1994;371(6499):707-711.

50. Rohrer SP, Jacobson EB, Hayes EC, Birzin ET, Schaeffer JM. Immunoaffinity purification of avermectin-binding proteins from the free-living nematode Caenorhabditis elegans and the fruitfly Drosophila melanogaster. Biochem J. 1994;302(Pt 2):339-345.

51. Dent JA, Smith MM, Vassilatis DK, Avery L. The genetics of ivermectin resistance in Caenorhabdwitis elegans. Proc Natl Acad Sci U S A 000;97(6):2674-2679

52. Gloeckner C, Garner AL, Mersha F, et al. Repositioning of an existing drug for the neglected tropical disease Onchocerciasis. Proc Natl Acad Sci U S A. 2010;107(8):3424-3429.

53. Boussinesq M, Gardon J, Gardon-Wendel N, Kamgno J, Ngoumou P, Chippaux JP. Three probable cases of Loa loa encephalopathy following ivermectin treatment for onchocerciasis. Am J Trop Med Hyg. 1998;58(4):461-469.

54. Addis DG, Rheingans R, Twum-Danso NAY, Richards F. A framework for decision-making for mass distribution of Mectizan ${ }^{\circledR}$ in areas endemic for Loa loa. Filaria J. 2003;2 Suppl:S9.

55. Duke BO, Zea-Flores G, Castro J, Cupp EW, Munoz B. Comparison of the effects of a single dose and of four six-monthly doses of ivermectin on adult Onchocerca volvulus. Am J Trop Med Hyg. 1991;45(1): $132-137$.

56. Chavasse DC, Post RJ, Lemoh PA, Whitworth JA. The effect of repeated doses of ivermectin on adult female Onchocerca volvulus in Sierra Leone. Trop Med Parasitol. 1992;43(4):256-262.

57. Sauerbrey M. The Onchocerciasis Elimination Program for the Americas (OEPA). Ann Trop Med Parasitol. 2008;102 Suppl 1:25-29.

58. Kyelem D, Medlock J, Sanou S, Bonkoungou M, Boatin B, Molyneux DH. Impact of long-term (14 years) bi-annual ivermectin treatment on Wuchereria bancrofti microfilaraemia. Trop Med Int Health. 2005; 10(10):1002-1004.

59. Diawara L, Traore MO, Badji A, et al. Feasibility of onchocerciasis elimination with ivermectin treatment in endemic foci in Africa: First evidence from studies in Mali and Senegal. PLOS Negl Trop Dis. 2009; 3(7):e497. 
60. Hoerauf A, Volkmann L, Hamelmann C, et al. Endosymbiotic bacteria in worms as targets for a novel chemotherapy in filariasis. Lancet. 2000;355(9211):1242-1243.
61. Wanji S, Tendongfor N, Nji T, et al. Community-directed delivery of doxycycline for the treatment of onchocerciasis in areas of co-endemicity with loiasis in Cameroon. Parasit Vectors. 2009;2:39.

\section{Publish your work in this journal}

Research and Reports in Tropical Medicine is an international, peerreviewed, open access journal publishing original research, case reports, editorials, reviews and commentaries on all areas of tropical medicine, including: Diseases and medicine in tropical regions; Entomology; Epidemiology; Health economics issues; Infectious disease; Laboratory science and new technology in tropical medicine; Parasitology; Public health medicine/health care policy in tropical regions; and Microbiology. The manuscript management system is completely online and includes a very quick and fair peer-review system. Visit http://www.dovepress. com/testimonials.php to read real quotes from published authors.

Submit your manuscript here: http://www.dovepress.com/research-and-reports-in-tropical-medicine-journal 